\title{
¿POR QUÉ GRECIA, TODA GRECIA? (UN ENSAYO DE ENFOQUE INTERCULTURAL SOBRE DOS FORMACIONES IDENTITARIAS)
}

\author{
María Amoretti Hurtado
}

\begin{abstract}
RESUMEN
Luego de participar en el Primer Congreso de Neohelenistas de Iberoamérica, la autora presenta sus investigaciones sobre el cuento griego moderno y sobre los estudios neohelénicos en Latinoamérica.
\end{abstract}

\begin{abstract}
After participating in the First Ibero-American Neohellenian Congress, the author presents her research on modern Greek short story and on Neohellenian studies in Latin America.
\end{abstract}

\section{Primer congreso de neohelenistas de Iberoamérica}

Por gentil invitación de la Embajada de Grecia en Madrid, cursada por intermedio del Decano de la Facultad de Letras de la Universidad de Costa Rica, Máster Enrique Margery Peña, la licenciada Estébana Matarrita representó a nuestro país en tan importante encuentro. La licenciada Estébana Matarrita pertenece al Departamento de Filología Clásica de la Escuela de Filología, Lingüística y Literatura y ha sido encargada de la Cátedra de Griego Clásico por muchos años.

Este Congreso, llevado a cabo la última semana del mes de febrero de 1996, es la culminación de los esfuerzos del profesor Moschos Morfakidis, promotor incansable de la cultura neohelénica en la Universidad de Granada, España, en donde tuvo lugar este convivio. Desde allí, gracias a sus diligencias, se ha convocado por sexta vez a los especialistas de la cultura griega moderna. En esta ocasión, sin embargo, esa reunión se proyectó fuera de Europa, incluyendo además a la comunidad académica de América Latina, de ahí su importancia para nosotros. De nuestro continente, además de Costa Rica, estuvieron representados los siguientes países: Argentina, Colombia, Brasil, Chile y México.

Por simple interés personal, decidí acompañar a la colega Matarrita y presentar una ponencia sobre el cuento griego moderno desde un enfoque intercultural frente a los discursos identitarios que el desarrollo del cuento costarricense manifiesta. El propósito del presente artículo es el de compartir con los lectores de la Revista de Filología y Lingüística esa comunicación, al 
tiempo que establecer una especie de estado de la cuestión respecto de los estudios neohelénicos en el continente, información que me fue posible recoger durante la actividad gracias al fructífero intercambio con colegas de Latinoamérica. Pienso que el interés de estos datos estriba precisamente en que este campo, el del neohelenismo, es un sector académico bastante descuidado en nuestra región y Costa Rica no es una salvedad. No obstante, ha sido la Universidad de Costa Rica, la única institución que en este país se ha interesado en introducirlo mediante la Escuela de Lenguas Modernas y los cursos de Griego Moderno que en esa Unidad Académica se han ofrecido. Desgraciadamente, estos esfuerzos han sido esporádicos, sin línea de continuidad. El desarrollo de esta área del saber ha estado marcada además por ciertas condiciones de emergencia que se han repetido en todas aquellas Universidades que la han introducido. Esto es que, con muy pocas excepciones, los estudios neohelénicos surgen normalmente en el seno de los departamentos de Estudios Clásicos y no logran la independencia necesaria para plantearse como un proyecto que tiene objetivos propios y su objeto continúa opacado por la relevancia histórica de la Grecia Clásica y su cultura. Este fenómeno fue particularmente interesante en las discusiones mismas de este Primer Congreso de Neohelenistas de Iberoamérica. La pregunta esencial volvía una y otra vez en las diferentes ponencias: “¿Entre la Cultura Griega Clásica y la Cultura Griega Moderna, hay o no hay una relación de continuidad?”. Las respuestas nunca fueron intermedias, los estudiosos se afiliaban por lo general a un sí o a un no sin matizaciones. Como se puede colegir, el planteamiento de esa pregunta significaba un esfuerzo por establecer un objeto formal que justificara la autonomía de un sector en el campo intelectual: la cultura neohelénica. Esta pregunta se discutía muy a menudo a partir de la relación entre las dos lenguas, a partir del fenómeno linguiístico en sí, subyaciendo, sin embargo, que de las conclusiones que de ello se extrajeran, se podía derivar una respuesta aplicable también al hecho cultural como un todo. También para encontrar respuestas a esa pregunta, fue que dicidí participar en ese congreso. Pero en nuestro caso, había preguntas anteriores a esa que débían ser previamente planteadas y respondidas, como lo veremos más adelante.

En la formación de todo filólogo (y yo lo soy), como en la de cualquier otro humanista, Grecia Clásica ocupa un papel preponderante, y su historia ilumina el origen de nuestra civilización occidental. Desafortunadamente, en nuestros planes de estudios, como en muchos otros currículos de otras instituciones del continente, la historia hace un corte salvajemente radical y la Grecia de nuestros libros de griego y literatura clásica desaparece en las postrimerías del siglo XIII. El vacío que se crea no atina a encontrar un nexo que reúna a aquella Grecia con la realidad palpitante de la Grecia de nuestros días.

Cuatrocientos años de dominación otomana, más los avatares bélicos y políticos que se prolongan hasta las dos guerras mundiales y más, median en esa transformación. Viendo, pues, la oportunidad de llenar ese vacío, de tratar de comprender el destino de este pueblo, me fui para Granada en compañía de la profesora Matarrita. Durante tres días de jornadas de doce o catorce horas de trabajo diario, asistimos a un acontecimiento académico de gran envergadura. Más de cien ponencias que abarcaban las metodologías en la enseñanza del griego moderno, los problemas de traducción y transcripción de la grafía griega, la literatura griega contemporánea, hasta las imágenes de Grecia en las guías turísticas europeas, me dieron un panorama no sólo enriquecedor sino también tan excitante e incitante que en lugar de aplacar mi curiosidad, la ha aumentado. 
Decíamos que la Grecia Clásica ha obnubilado siempre el interés por la Grecia Moderna. Cuando el visitante llega a Grecia, generalmente se preocupa sólo por las obras maestras clásicas o por los monumentos de la época bizantina (dos de los períodos más grandes del arte en Grecia) y elide la existencia del arte griego contemporáneo, el cual puede no alcanzar las cumbres logradas por la Atenas del siglo V A.C., o por la Constantinopla del siglo X D.C., pero es un arte que entronca sustancialmente en la misma tradición. La anterior afirmación es la conclusión a la que llegamos a través del examen que efectuamos del desarrollo del cuento griego moderno, como se verá más adelante.

Así, los conceptos de continuidad o ruptura entre las dos Grecias, serían una constante en muchas de las comunicaciones del Congreso. La forma en que las diferentes posiciones se justificaron, es un comentario que dejaremos también para su momento oportuno.

La presencia de Su Majestad, Doña Sofía, la Reina de España y su hermana, le dio al Congreso una dimensión muy especial y el auspicio de la Embajada de Grecia en España, aportó a los congresistas no sólo abundante información y apoyo bibliográfico, sino toda clase de material audiovisual para los profesores del cultura neohelénica. Recientemente, además, desde Grecia, la Facultad de Letras recibió tres bultos de material bibliográfico de excelente calidad tanto por su contenido como por la calidad de las ediciones, que el señor Decano ha puesto ya en disposición de los usuarios en la Biblioteca de la Facultad. Uno de los logros más importantes del Congreso fue la creación de la Sociedad Hispánica de Estudios Neogriegos, cuyos estatutos se aprobaron en el marco de la hermosa ciudad de Santa Fe.

\section{Estudios neogriegos en Hispanoamérica. Estado de la cuestión}

Como ya dijimos, en Costa Rica, la relación con la cultura neohelénica es más que limitada, pobre. Basta al respecto un recuento de lo que en relación con ella ofrecen nuestras bibliotecas, en las que la misma clasificación confunde la Grecia Clásica con la Moderna, bajo la rúbrica de Grecia, es la Clásica la que domina el panorama, con el mayor agravante de que en el sistema automatizado se confunden las referencias bibliográficas entre la Grecia europea y la Grecia de Alajuela. De los 322 títulos con que contamos en ese apartado, la mayoría se refieren a la Grecia cañera.

La mayor limitación está, no obstante, en la traducción y a esto es a lo que me voy a referir seguidamente, muy concretamente en el caso de Hispanoamérica.

Tres países de Hispanoamérica se destacan por su vivo interés en la cultura griega moderna: Argentina, Chile y Venezuela. Pero de los tres, es Chile el que tiene en este interés una tradición académica más prolongada y una organización institucional más consolidada.

En Chile, al igual que en la mayoría de las comunidades intelectuales de otros países, es la preocupación helénica la que sirve de base inicial y motora para el desarrollo posterior de los estudios neohelénicos. En este sentido, Costa Rica y México son la excepción, pues en estos países son los departamentos de lenguas modernas los que han albergado los estudios neohelénicos, nos referimos al caso de la Universidad de Costa Rica (UCR) y a la Universidad Nacional Autónoma de México (UNAM).

En Chile, al igual que en España, es a partir del desarrollo de los estudios helénicos, que se llega a la preocupación por lo neohelénico. La Biblioteca de Chile jugó en ello un papel 
muy decisivo gracias a los títulos que al respecto importaba de países como Alemania y Francia, por ejemplo. Poco a poco este interés se fue consolidando con la apertura de cursos sobre el mundo bizantino y panoramas de la literatura neohelénica hasta que gracias a estos impulsos se llega a la creación de un centro de estudios bizantinos en la Facultad de Filosofía y Humanidades de la Universidad de Chile, en 1967, precedida ya por una extensa lista de traducciones publicadas en ese país, la cual se remonta desde el período colonial con traducciones de las obras maestras clásicas hasta las traducciones de obras literarias de la Grecia Moderna en este siglo, como las traducciones de obras de Kazantzakis, Elytis, Seferis y otros grandes escritores neogriegos, a partir de 1937, como se detallará más adelante.

\section{El centro de estudios bizantinos y neohelénicos "Fotios Malleros"}

La creación de este centro se debe a la labor del profesor Fotios Malleros, cuyo amor e incansable difusión de la cultura neohelénica desde los años cuarenta, deja como herencia discípulos preclaros tales como el profesor Miguel Castillo Didier y la fundación del Centro; así, en su honor, esta unidad académica lleva su nombre: Centro de Estudios Bizantinos y Neohelenísticos Fotios Malleros, al que de ahora en adelante nos referiremos por la sigla CEBN.

En virtud de la labor de investigadores como el ya citado Castillo Didier, y de otros como Alejandro Zorbas D. (actual Director del CEBN) y Héctor Herrera Cajas (acaso el único experto en el campo bizantino en América Latina), el CEBN se convierte, gracias a sus publicaciones, en una antena que convoca a todos los amantes de la cultura neohelénica en Hispanoamérica.

Se pueden contar veinticuatro publicaciones, entre las que podemos destacar las siguientes, que repertoriamos en orden cronológico, con el fin de que se observe la continuada y sostenida actividad que en investigación, traducción y publicaciones mantiene el CEBN:

Castillo Didier, Miguel. 1971. Antología de la literatura neohelénica. I Poesía. Santiago: Editorial Andrés Bello.

Herrera Cajas, Héctor. 1972. Las relaciones internacionales del Imperio Bizantino durante la época de las grandes invasiones. Santiago: Editorial Universitaria.

Stratigopoulos, Danae. 1972. Cantos de los griegos. Santiago: Editorial Universitaria.

Jaris, Petros. La noche larga. 1974. Introducción, traducción y notas por Fotios Malleros K. Santiago: Editorial Universitaria.

Panayotopoulos, I.M. 1978. Chipre, tierra del amor. Introducción, traducción y notas por Miguel Castillo Didier. Prólogo de Fotios Malleros. Santiago: Editorial Universitaria.

Kazantzakis, Niko. 1978. Teatro. Introducción, traducción y notas por Miguel Castillo Didier. Prólogo de Fotios Malleros. Santiago: Editorial Universitaria. 
1981. La espiritualidad griega y cristiana. Santiago: Alfabeta Imp.

Malleros, Fotios. 1987. El Imperio Bizantino 395-1204. 2da. edición revisada, aumentada y actualizada. Santiago: Editorial Universitaria.

Castillo Didier, Miguel. 1988. Las odas griegas de Andrés Kalvos. Ensayo y versión métrica completa. Santiago: Editorial Universitaria.

Tovar, Saúl A. 1990. Biografía de la lengua griega. Sus 3000 años de continuidad. Santiago: Editorial Universitaria.

Castillo Didier, Miguel. 1991. Kavafis íntegro. Santiago: Editorial Universitaria. Dos volúmenes.

Anghelids-Spinedi, Nina. 1992. Poetas griegas contemporáneas 1930-1990. Santiago: Editorial Universitaria.

Yanes Ritzos. 1993. Forma de la ausencia, Sonata Claro de Luna, Otros poemas. Selección, traducción, prólogo de Miguel Castillo Didier. Santiago: Editorial Universitaria.

Castillo Didier, Miguel. 1994. Poesía heroica griega. Epopeya de Diyenis Akaitas. Santiago: Editorial Universitaria.

Vakalópulos, Apóstol. 1995. Historia de Grecia Moderna. 1204-1985. Traducción y notas de Alejandro Zorbas y Nikiforos Nuikolaides. Santiago: Editorial Universitaria.

También en este último año se ha publicado un novedoso estudio sobre Francisco Miranda titulado: Francisco Miranda y Grecia. Precursor, héroe y mártir de la independencia hispanoamericana. Bellísima edición, obra de Miguel Castillo Didier, en la que la figura de Miranda, precursor de la libertad hispanoamericana, representa el espíritu griego absorbido en la cultura clásica griega, el cual se convierte en práxis moderna que él ha de heredar como ideal a otros próceres del continente tales como Bolívar, Bello y Martí. A través del pensamiento de Miranda y su vida, Castillo Didier rastrea el espíritu de la Hélade en las raíces de la historia hispanoamericana.

Aparte de esta extensa y enjundiosa lista de ediciones, el CEBN cuenta además con una publicación periódica: Bizantion Nea Hellás, única revista especializada en América Latina en el campo neohelenístico.

Justo es destacar que la extraordinaria labor desplegada por el CEBN ha sido apoyada incansablemente por los generosos aportes anuales que desde 1968 ininterrumpidamente vienen haciendo los sucesivos gobiernos de Grecia, a los cuales habría que abonarles también su interés por la preparación de personal especializado en el campo de la neohelenística en Hispanoamérica. Así, tenemos el caso del profesor Saúl A. Tovar, becado por la Universidad Aristotélica de Salónica y por el Ministerio de Relaciones Exteriores de Grecia, diplomado en Lengua Griega 
Moderna en la Universidad de Atenas, este argentino, además doctor en Filosofía por la Universidad de Córdoba, es también colaborador del CEBN.

Está también el caso de Pedro I. Vicuña que estudió, becado por el gobierno de Grecia, en la Escuela de Arte Dramático del Teatro Nacional de Grecia. Vicuña es también diplomado en Lengua Griega por el Instituto Manoli Triandafillidi, de la Universidad de Salónica. Entre sus muchas obras, vale la pena citar su traducción al castellano de una antología de la poesía de Yorgos Seferis y otra de la poesía de Odysseas Elytis, comprada esta última por Alianza Editorial de España.

\section{El reconocimiento de la filología bizantina y neogriega como área de conocimiento}

Ahora, con la creación de la Sociedad Hispánica de Estudios Neogriegos se oficializa el reconocimiento de la Filología Bizantina y Neogriega como área de conocimiento.

Los estatutos de este organismo al que, por afiliación de dos de sus profesores, se relaciona ahora la Universidad de Costa Rica, le dan como propósitos no sólo la promoción de los estudios neogriegos, sino también la defensa y consolidación de su reconocimiento como campo de estudios independiente. Así, entre las funciones de la SHEN (Sociedad Hispánica de Estudios Neogriegos) está la de proponer a las instituciones públicas o privadas proyectos tendientes a la incorporación de los estudios neogriegos en los planes de enseñanaza e investigación.

La sede de la SHEN quedó establecida oficialmente en la Facultad de Filosofía y Letras de la Universidad de Granada y la preside actualmente el profesor Moschos Morfakidis.

Después de este extenso pero necesario preámbulo, transcribimos a continuación la ponencia que con ocasión del Primer Congreso de Neohelenistas de Iberoamérica presentamos en la Comisión dedicada a la Literatura Griega Moderna; sin embargo, dado que el planteamiento esencial de mi trabajo era un postulado cuestionado explícita o implícitamente en algunas de las exposiciones del congreso, aunque defendido y mantenido por otros autores, debo nuevamente hacer una digresión, para contextualizar mis ideas y justificar la legitimidad de mis inquietudes.

Como lo expuse al inicio de este artículo, los motivos para participar en este convivio tenían su origen en mi propia formación de filóloga, en la que el papel de la Grecia Clásica es uno de los pilares fundadores; pues bien, en esa formación académica ningún corte histórico es tan radical -decíamos- como el que se hace entre la Grecia Clásica y la Grecia moderna. Es tan radical este corte que el pasado de este pueblo no pareciera tener línea de continuidad con el presente. ¿Qué ocurrió en ese vacío histórico? ¿Cuánto de la Antigua Grecia y su esplendor sobrevive en la Grecia de hoy? Eran las preguntas que yo quería contestar participando en el Primer Congreso de neohelenistas de Iberoamérica. Mi sorpresa fue que los grandes especialistas que allí se convocaron no tenían para esas interrogantes una respuesta unívoca.

Dos tesis muy radicales afloraron. Ruptura o continuidad fueron una especie de supuesto fundador sobre el que se sostuvieron algunas de las exposiciones que se presentaron.

Mientras Saúl A. Tovar sostiene en su libro Biografia de la lengua griega. Sus 3000 años de continuidad que ha habido una contigüidad histórica y una continuidad lingüística entre ambas Grecias; la comunicación presentada por Pedro Bádenas de la Peña, por ejemplo, exponía 
la disglosia bizantina como un fenómeno literario en tanto que en la Grecia moderna es cuestión de política educativa y tiene su propio origen, fines y mecanismos (se refería al lenguaje hablado o demótico y a la lengua aticista, llamada katharévusa). Pero, además y en términos generales, exigía una necesaria separación entre la Grecia Clásica y la Moderna y señalaba la conveniencia de superar la idea de "una civilización unitaria".

Hablaba también de la necesidad de revisar lo que él llamaba "algunos mitos conceptuales". Entre esos mitos se refería al de Bizancio como "mero transmisor y conservador del mundo antiguo" y señalaba que entre ellos existió un abismo tan grande que más bien debería percibírseles como mutuamente excluyentes, pues el helenismo y el bizantinismo se repelían de la misma forma en que se repelían el paganismo y el cristianismo y que no fue sino después de 1204 que algunos autores bizantinos se sintieron más identificados con lo clásico. Pero también aclaraba Bádenas que ambas culturas se amalgaman en el siglo XIX en el momento en que comienza la formación de una conciencia nacional. Y esta última afirmación me tranquilizó bastante, pues de algún modo dejaba todavía en pie lo sustancial de mi ponencia.

Por eso, nos parece, que los argumentos de Bádenas iban más bien en el sentido de que la superación de lo que él llamaba "mitos conceptuales" son una necesidad para poder diseñar una estrategia política adecuada tendiente a legitimar la existencia del griego medieval y moderno como campos académicos autónomos. La ruptura que Bádenas planteaba era más bien la de la autonomía de la Filología Neogriega en relación con el papel ancilar que históricamente ha mantenido en relación con la Filología Clásica en el seno de las instituciones universitarias, especialmente en Europa, muy particularmente en España e igualmente en el caso de Chile, como ya lo vimos.

De modo que, preguntarse, como lo hacía mi ponencia, sobre la forma en que había evolucionado el patrimonio de un pueblo -el de la Grecia Clásica, convertido por lo demás en patrimonio universal-, dentro de la misma comunidad que le dio origen, continuaba siendo una interrogante legítima y un tema de meditación pertinente. El campo en donde iba a buscar los índices de una posible continuidad tenía que ser dentro de la literatura, como el campo de la especialidad a la que me he dedicado por mucho tiempo.

\section{El cuento griego moderno y el cuento costarricense (un ensayo de enfoque intercultural entre dos formaciones identitarias)}

Dentro del vasto territorio de la literatura neogriega, seleccioné el cuento por dos razones de necesidad, las cuales hacen prácticamente que en realidad yo no seleccioné el cuento, sino que el cuento griego me seleccionó a mí.

La primera de esas razones es mi interés por el relato corto en general. Desde hace casi una década he estado dedicada a su estudio, particularmente a la investigación del relato corto en Costa Rica, su origen y su evolución. Mi interés en el cuento costarricense tiene como objetivo esencial la indagatoria de los procesos identitarios en mi comunidad.

La segunda razón es un hecho fortuito y ligado a la pobreza de fuentes bibliográficas sobre literatura neohelénica en Costa Rica. Mi texto de base es una antología preparada y editada por el Centro de Estudios Bizantinos y Neohelénicos Fotios Malleros, de la Universidad de Chile, con la colaboración de algunos especialistas que hoy nos acompañan: Miguel Castillo 
Didier y Saúl A. Tovar. Dicho texto llegó casi que azarosamente a mis manos. Se lo vi a la colega Matarrita, quien lo recibió como un obsequio de parte de Ana Passanidis -que también hoy está con nosotros en este convivio- durante un Congreso de Helenistas en Buenos Aires, en 1989. Ana Passanidis es precisamente la Directora del Instituto Griego de Cultura en Argentina. Así llegó el cuento griego moderno, versión española, a Costa Rica y a mis manos. Esta antología recoge muestras cuentísticas desde el origen de la literatura neogriega hasta la década de los sesenta y mostraba coincidencias cronológicas en dos literaturas nacionales que tienen la misma edad. La lectura de esta antología me permitió una panorámica del género, sus tendencias principales, sus escuelas y su evolución. Mis elucubraciones al respecto no deben ser tomadas más que como planteamientos provisionales, casi aleatorios, que deberán ser confirmados posteriormente, conforme adquiera mayor trayectoria en las veredas de la literatura neohelénica. No obstante, me complace exponerlos (con todo el sentido de riesgo que la palabra "exponer" conlleva), ante las autoridades por cuyo criterio y conocimiento se elaboró la misma antología de la que me he servido.

Para comenzar, podríamos decir que la presencia de la Grecia Clásica en mi país se evidencia de inmediato. Basta una mirada a cualquier mapa político, para encontrar, aunque rodeadas de cañales o cundidas de frutas tropicales, las ciudades de Grecia, Atenas y Esparta: Pero estos índices helénicos en nuestra toponimia, podrían tomarse como meras curiosidades, comparados con el alcance que el espíritu de la Hélade tiene en la construción de una imagen nacional en Costa Rica, ya que es en la formación de su idiosincrasia en donde este espíritu resulta más significativo. La imagen que el país se da a sí mismo y por medio de la cual identifica culturalmente a sus sujetos, es la de una comunidad profundamente democrática. Ser la democracia más antigua de Hispanoamérica es el ícono que ofrece Costa Rica al exterior para su circulación. Pero a diferencia de otras democracias, la democracia costarricense se entiende más bien como "ágora"; es decir, como aquella en la que la idea de igualdad se define más que como una igualdad de oportunidades, como una igualdad en la posesión de la palabra; libertad es ante todo para el costarricense, su libertad de expresión, sólo por eso cree vivir en democracia.

Dados mis propios intereses personales en esta línea de investigación sobre la formación discursiva de la identidad en Costa Rica, mi encuentro con el cuento griego moderno está condicionado por esa misma preocupación. Mi punto de referencia es pues, el de mi espacio más familiar: la propia literatura nacional, la literatura de Costa Rica, su historia y su discurso identitario.

1821 es tanto para Grecia, como para Costa Rica, una fecha clave, liminar, pues para ambas comunidades representa el símbolo de su independencia. El mismo tiempo nos separa obviamente de esa fecha y, sin embargo, para los costarricenses el año no tiene gran relevancia. El 15 de septiembre es el día de la independencia, pero 1821 es un dato que no todos los costarricenses guardan en su memoria. Para Grecia, en cambio, esa fecha genera en su práctica lingüística toda una serie de fenómenos de resemantización y lexicalización de variada índole. Basta decir el 21, los del 21, para traer a colación la escena de las luchas independentistas. El 21 es sinónimo de lucha y los del 21 son los luchadores. La carga semántica e ideológica del 21 es fundadora de toda una práctica discursiva que se inscribe en sus textos literarios, como lo pude observar. 
La impronta que los acontecimientos de la guerra de independencia dejan en la práctica discursiva de la comunidad, está muy patente en estos cuentos en los que la historia y la ficción se unen para remontarse a la época de la liberación. Tal es el caso del relato titulado "La Patria" de Andreas Karkavitsas o "Las preciadas armas" de Giannis Vlajogiannis. Pero también está muy presente en aquellos relatos en los que el trabajo poético de metaforización elevan la experiencia humana y familiar de la guerra a la dimensión trágica del dolor y la mutilación, como es el caso de los cuentos "Una madre" de Thanasis Petsalis Diomidis, "Las Gaviotas” de Illías Venezis y "Regalo de una noche" de Tatiana Stavrou.

El 21 genera un discurso fundador que testimonia un fuerte sentido épico en el proceso identitario, heroísmo al que no escapan ni las mujeres ni los niños, ni los amantes.

Caso contrario es el de Costa Rica, porque este es el caso de un país sin épica. En 1821 la independencia asombra y asusta a la comunidad costarricense porque la toma por asalto. Pacíficamente vivieron durante la colonia española, ignorados y olvidados para su bien de todo el mundo y pacíficamente reciben también su independencia, casi que por rebote, como efecto de las luchas independentistas llevadas a cabo por otras provincias del Imperio tanto en el norte como en el Sur del continente. 1821 es para el caso de Grecia tan sólo el inicio de una lucha difícil que se ha de prolongar por muchos años envuelta en sangre, actos heroicos y traidores. 1821 es el año en que un grito de autonomía prolongado se va a oír constantemente enardecido o sofocado, mientras Grecia se va recuperando pedazo a pedazo.

Así, mientras en la literatura griega la figura del héroe se va a constituir en una figura emblemática, en la literatura costarricense la figura del campesino va a dar cabida a la ambivalencia de un héroe ironizado que termina emergiendo en la difusa imagen de la victimización.

Mientras en el cuento griego el proceso de subjetivación, de la constitución de un sujeto cultural, se ve fuertemente ligado a la figura del héroe, a los procesos de heroificación y a la comprobación de la falsedad o autenticidad de los héroes; en la literatura nacional de Costa Rica la figura de la víctima asume el papel de sujeto cultural y desencadena un proceso de identificación sumamente conflictivo con la imagen nacional. El cronotopo de la víctima desarrolla, para los efectos de la constitución de un sujeto cultural, una relación sumamente compleja con una emblemática en la que se funden ambivalentemente la necesidad y la virtud, ya que el campesino, ícono del nacionalismo costarricense, hace de la pobreza y la humildad, un heroísmo sin épica; hace de él un héroe que los mismos discursos literarios tratan de manera irreverente o compasiva, aun cuando tengan la intención explícita de cantar loas en su honor.

En el cuento griego, por el contrario, aún en los casos en que el desarrollo del relato cuestione la figura del héroe auténtico, la diégesis rescata el heroísmo como valor absoluto. Así, en el cuento "La Patria" de Andreas Karkavitsas, dos veteranos de la guerra de la independencia se encuentran muchos años después y comparando sus recompensas, el verdadero héroe está a punto de ser convencido de que su imagen de héroe no es más que el reverso del de la víctima de una entrega tonta e idealista:

El rumeliota se perturbó. En las impetuosas palabras de su amigo vio arrastrados todos sus sentimientos, igual que la caída del agua desarraiga y machaca los culantrillos de la roca. Y en el lugar de aquellos sintió que brotaba, molestándolo, una nueva interrogante, ¿Qué es mejor, la Patria o la persona? He aquí él y he aquí también su amigo. Este está reposando sobre los esfuerzos de su juventud (...) Y él, que no dedicó ni 
una hora al servicio de sí mismo, vagabundea ahora sin nada, despreciado por la gente... (El cuento griego moderno 1989: 42-44)

Pero en el momento final contraponiendo al interminable listado de beneficios personales que Lambrópoulos enumeraba muy elocuente, Petroletsos reacciona y en una imagen que como repentina visión se le aparece ante sus ojos encuentra la retribución a su holocausto:

...vio a la joven mirándolo a los ojos y mostrando con su mano la lejanía. Atisbó entonces Petroletsos y observó un país grande. Y adivinó inmediatamente que era Grecia, libre de punta a cabo, gloriosa, brillantemente iluminada (...) Saltó arrogante, severo, tal como en Keratsini luchaba contra los enemigos por el estandarte y al "¿qué ganaste?" que le preguntó el moreota:

- ¡Hice a mi Patria!, le espetó, golpeando sin compasión su pecho.

(El cuento griego moderno 1989: 43-44)

En otros casos, como el del cuento "El funesto Capitán” de Zajarías Papandoníou, se sigue la lógica inversa: se castiga duramente la falsificación del héroe y se conmina a los culpables de la falsificación a la ridiculización social y a la condena moral; porque el héroe no es únicamente sinónimo de valentía y entrega desinteresada por los demás, sino también receptáculo de los más altos valores morales. Esta imagen integradora del bien en la figura del héroe es tratada por Giannis Vlajogiannis en "Las preciadas y honrosas armas", en el que un Capitán, veterano de la guerra de independencia, yace moribundo en medio de una lacerante pobreza, pero se resiste a vender sus brillantes armas para pagar un médico, porque esas armas no son suyas:

\footnotetext{
-Ya es tarde para que los reconozca; y le habría hecho bien. Tantos años que habla de ustedes.

- ¿Le habló sobre esto?, preguntó Ziguris sorprendido.

-Me lo dijo todo... ¡cuánto se arrepintió!; dejó orden de ponerle las otras, sus armas pobres, a su muerte. En los últimos días me indicó que descolgara esas (y mostró las armas ricas botadas sobre la mesa), las escondiera y le escribiese a usted en cuanto tuviera cómo hacerlo.

Había dirigido la vista hacia abajo y temblaba de turbación, pero nunca habría podido sacar una lágrima de sus ojos.

-No diga estas cosas, contestó Ziguris; buscaremos la manera de vender las armas para que usted atienda al enfermo.

- ¿Me hizo jurar, las armas le pertenecen a tu señoría! es su postrer encargo.

(El cuento griego moderno 1989: 67)
}

El orgullo del héroe no se apaga por la ausencia de grandes retribuciones y los altos valores que encarna sostiene su especial condición, la cual es igualmente proyectada por su mujer quien también actúa "heroicamente".

Todo lo contrario, en las representaciones colectivas del imaginario en Costa Rica, la reivindicación del campesino como héroe nacional está siempre matizada, aunque sea a nivel implícito, de cierto cuestionamiento, como es el caso de los cuentos de Magón uno de los escritores del nacionalismo literario de finales del siglo pasado y su transformación mórfica hereda, de representación en representación, su condición de víctima. Así lo constata Amalia Chaverri en el análisis que hace sobre la titulología de nuestra narrativa mayor (la novela) en la que perfila una metamorfosis del desarraigo hasta la pauperización y la conversión del campesino en paria urbano. 
Por otro lado, al comparar la imagen de la patria ofrecida por el cuento de Karkavitsas con la imagen que se da en la simbólica patria costarricense, hay una diferencia muy radical. En la letra del Himno Nacional de Costa Rica, por ejemplo, la patria es la figura materna de la que el hijo se alimenta casi que por ley natural y está más ligada a unos topoi arcádicos que la anclan al imaginario colectivo como una categoría a priori, que antecede al sujeto y a la que éste está obligado a defender como imposición de deber filial. Por el contrario, la patria de Petroletsos es una patria forjada por él, creada por el sujeto, nacida de sus manos para su propia redención, por lo que se evidencia un proceso de identificación no problematizado. La Patria es la proyección del héroe.

Esa es una de las razones por las que si bien en ambos cuentos, el griego y el costarricense, observo un fuerte anclaje referencial, la función de esa referencialidad es bien diferente. En el griego ese anclaje parece venir de una historia traumática y difícil de ignorar, mientras que en el costarricense parece derivar de una relación especular de naturaleza ideológica entre el hombre natural que es el campesino y su hábitat.

No obstante, ese efecto de naturalidad, o de sencillez -como se le ha llamado en nuestro país-, se invierte en el plano discursivo de las manifestaciones estéticas. Mientras en el cuento griego moderno se capta una relación directa, sin escamoteo, entre el narrador y el lector, en el cuento costarricense (y en la novela también) la modalidad irónica es preferencia discursiva por excelencia, ella marca nuestros relatos desde su origen y se mantiene como signo esencial a lo largo de todo el desarrollo de la narrativa costarricense. Las preferencias discursivas y la puesta en escena de la intencionalidad de los relatos costarricenses ponen de manifiesto que la sinceridad y la verdad textual son difícilmente accesibles en sus niveles explícitos, consecuencia posible de los conflictos identificatorios con el sujeto cultural.

Lo mismo ocurre con sus desenlaces, punto esencial en la estructura del género. El modelo griego no favorece, en general, los finales abruptos, el devenir del relato se desliza con naturalidad hacia su final.

Hay una preocupación ética que se evidencia en el cuento griego no sólo en la modalidad enunciativa, sino también en las variadas formas en que la temática se figuraliza o argumenta; lo que hace -a mi juicio- que los acontecimientos pasen a segundo plano -a pesar de los elementos referenciales- para dejar en escena las consecuencias emotivas y los fenómenos de conciencia que se dan en torno a ciertos valores y a una cierta moralidad; todo, en la relación intersubjetiva. En ese aspecto es paradigmático el cuento "El orgullo de la salud" de Nikos Nikolaídis y también "La última noche de la tierra" de Petros Jaris.

En el primero, se relata el lento proceso de la incubación del odio como producto de un estímulo externo que resulta insignificante si lo comparamos con las elaboraciones que la conciencia hace a partir de él. El inicio del relato muestra cómo el personaje-narrador, que no hace otra cosa que dialogar consigo mismo, va elaborando un estado de ánimo positivo hasta llevarlo a la euforia; pero luego un encuentro casual, con un desconocido cuya imagen es el oximoron perfecto de la razón de su gozo, genera un proceso inverso que lo hace involucionar hasta el estado de ánimo contrario: la repulsión y el odio, para terminar destruido por una monstruosa morbilidad de conciencia. Compárense dos fragmentos que representan los dos estados de ánimo de cuya transformación da cuenta el relato: 
Estaba feliz feliz llevaba en mí todas las bondades y no podría hacer sufrir a nadie. Así, alguien podría sólo mirarme y comprendería la bondad que provenía de mi salud y mi buen ánimo (...)

Tenía la sensación de que caminaba feliz entre gente feliz...

La ola dorada de la vida fluía en mis adentros...

De pronto el arrancado de la eutanasia espartana, se apareció adelante mío para desnudarme, no sólo de mi orgullo y de la poesía de mi juventud, sino que, además, de mi propio honor" (106).

Esta es la visión de un inválido cuya riqueza le permite ser un enfermo soberbio, según el punto de vista del personaje-narrador, y esta contrariedad al orgullo de la salud que él siente, causa en él el nacimiento de una semilla de odio que lo lleva hasta intentar eliminar al inválido, para hacer prevalecer su idea de que la salud es motivo de orgullo. Fracasa en un intento en el que la víctima ni siquiera se percata del peligro en que estuvo y el personaje narrador cierra el relato así:

Desde entonces la gente me mira y me considera como un hombre fracasado y disgustado que se descarga en los otros. Y el escapado de los abismos de Esparta continúa paseando por todas partes y delante mío su asqueroso cuerpo, desafiante y cínico. Intocable (117).

En el cuento "La última noche de la tierra" de Petros Jaris, una pareja de sirvientes condenados a sufrir en desamparo la hecatombe de la que sus patronos se han puesto a buen recaudo abandonándolos a ellos a la intemperie, logran construir en el lapso de esa larga noche -la última de la tierra- una comunión espiritual tan fuerte mediante el amor que logran que todo vuelva a renacer de nuevo.

Esta relación intersubjetiva se observa en muestras abundantes a todo lo largo de la historia del cuento griego, mientras que en el cuento costarricense es más propia de las narradoras femeninas y sólo se cristaliza a partir de los años cuarenta.

Si el helenismo ha sido el padre de sentido épico y el humanismo nace a partir del espíritu de la Hélade, no cabe pensar otra cosa que la preeminencia de la figura del héroe en el discurso literario del cuento griego y el interés por la sustancia anímica o espiritual de sus personajes frente al mundo y los acontecimientos, representa, al menos en la práctica literaria, una línea de continuidad entre la cultura de la Grecia Clásica y esta otra de la Grecia Moderna quien, al igual que los otros pueblos de la Civilización Occidental, la ha heredado como patrimonio, pero que, a diferencia de esos otros pueblos, la ha también sabido recuperar como horizonte de su propia construcción como comunidad y en la conciencia que de su unidad nacional ella se da a sí misma. De modo que, a nuestro juicio, es el nacimiento de una conciencia nacional el que recupera la tradición helénica en el momento en que la Grecia Moderna se forja como nación independiente y traza -sólo a partir de ese momento- una línea de continuidad con la Grecia Clásica.

El arte griego moderno es, entonces,la creación de un pueblo profundamente condicionado, para bien o para mal, por la experiencia de un extraordinario pasado artístico. Esto no quiere decir que no se evidencien en él las marcas de una historia tan convulsa como la suya y los avatares consecuentes en la formación de un sujeto cultural en el que alternan los signos de una tradición milenaria y los cambios que las precisas coyunturas históricas -bastantes turbulentas por cierto- obligan a efectuar en la formación de una conciencia nacional. Pero va a ser el espíritu de la Hélade, su sentido de la épica y la emblemática del héroe las marcas que en el cuento griego moderno van a manifestar las claves ideológicas sobre las que se va a sentar la creación de un orgullo nacional. 
Las reflexiones anteriores -provisionales y perentorias- son las conclusiones a las que llegamos a través de una primera aproximación con el desarrollo del cuento griego moderno, ayudados por un colimador intercultural como metodología de apoyo: la comparación con el desarrollo del mismo género en una comunidad que inicia en la misma fecha, aunque en circunstancias muy distintas, su vida independiente y la formación de un sujeto nacional.

\section{Nota}

Gran parte de la información ofrecida sobre la historia de los estudios neogriegos en Hispanoamérica se recolectó por medio del fructífero intercambio con colegas que tienen dentro de esta disciplina una experiencia y autoridad ya reconocidas. También se mencionaron detalles de las notas que la autora efectuó en el término de las sesiones a las que asistió.

\section{Bibliografía}

Castillo Didier, Miguel. 1995. Grecia y Francisco de Miranda. Santiago de Chile: Editorial Universitaria.

El cuento griego moderno. 1989. Antología publicada por el CEBN. Santiago de Chile: Editorial Universitaria.

Tovar, Saúl A. 1991. Biografía de la lengua griega. Sus 3000 años de continuidad. Santiago de Chile: Editorial Universitaria. 
